\title{
Ipsilateral motor pathways after stroke: implications for non-invasive brain stimulation
}

\author{
Lynley V. Bradnam ${ }^{1,2+}$, Cathy M. Stinear ${ }^{3,4}$ and Winston D. Byblow ${ }^{4,5 * t}$ \\ ${ }^{1}$ Brain Research Laboratory, Centre for Neuroscience, School of Medicine, Flinders University, Adelaide, SA, Australia \\ ${ }^{2}$ Effectiveness of Therapy Group, Centre for Clinical Change and Healthcare Research, School of Medicine, Flinders University, Adelaide, SA, Australia \\ ${ }^{3}$ Clinical Neuroscience Laboratory, Department of Medicine, The University of Auckland, Auckland, New Zealand \\ ${ }^{4}$ Centre for Brain Research, The University of Auckland, Auckland, New Zealand \\ ${ }^{5}$ Movement Neuroscience Laboratory, The University of Auckland, Auckland, New Zealand
}

Edited by:

Janine Reis,

Albert-Ludwigs-University Freiburg,

Germany

\section{Reviewed by:}

Robert Chen, Toronto Western

Hospital, Canada

Yoshikazu Ugawa, Fukushima Medical

University, Japan

\section{*Correspondence:}

Winston D. Byblow, Department of Sport and Exercise Science, The University of Auckland, Private Bag 92012, Auckland 1142, New Zealand. e-mail: w.byblow@auckland.ac.nz

${ }^{\dagger}$ Lynley V. Bradnam and Winston D. Byblow have contributed equally to this work.
In humans the two cerebral hemispheres have essential roles in controlling the upper limb. The purpose of this article is to draw attention to the potential importance of ipsilateral descending pathways for functional recovery after stroke, and the use of non-invasive brain stimulation (NBS) protocols of the contralesional primary motor cortex (M1). Conventionally NBS is used to suppress contralesional M1, and to attenuate transcallosal inhibition onto the ipsilesional M1. There has been little consideration of the fact that contralesional M1 suppression may also reduce excitability of ipsilateral descending pathways that may be important for paretic upper limb control for some patients. One such ipsilateral pathway is the cortico-reticulo-propriospinal pathway (CRPP). In this review we outline a neurophysiological model to explain how contralesional M1 may gain control of the paretic arm via the CRPP. We conclude that the relative importance of the CRPP for motor control in individual patients must be considered before using NBS to suppress contralesional M1. Neurophysiological, neuroimaging, and clinical assessments can assist this decision making and facilitate the translation of NBS into the clinical setting.

\section{Keywords: stroke, rehabilitation, upper limb, propriospinal, transcranial direct current stimulation}

\section{INTRODUCTION}

Reaching forward with the arm to manipulate objects with the hand is a quintessential function for higher order primates. Upper limb movements involve a fine balance between proximal stability and distal dexterity, presenting a unique motor control challenge to the central nervous system. There is a growing body of evidence that skilled upper limb function is under the control of both contralateral (cM1) and ipsilateral (iM1) motor cortices (Chen et al., 1997; Gerloff et al., 1998; Muellbacher et al., 2000; Hummel et al., 2003; Sohn et al., 2003; Verstynen et al., 2005; Davare et al., 2007; Duque et al., 2008; Perez and Cohen, 2008, 2009; Lee et al., 2010). Exactly how iM1 contributes to ipsilateral upper limb control is unclear, and is likely to involve both interhemispheric and descending projections. Neurophysiological studies have shown that $\mathrm{iM} 1$ assists $\mathrm{CM} 1$ to shape motor output by modulating the degree of transcallosal inhibition between homologous muscle representations in the two hemispheres (Sohn et al., 2003; Davare et al., 2007; Perez and Cohen, 2008). The potential importance of descending pathways from iM1 to spinal cord for upper limb control has largely been ignored. In this paper we present a novel hypothesis to account for how iM1 contributes to skilled upper limb motor control. We propose that the pathway involves a robust ipsilateral projection called the cortico-reticulopropriospinal pathway (CRPP), based on findings in the cat and non-human primate (Illert et al., 1981; Alstermark et al., 1984; Isa et al., 2006). The CRPP descends from iM1 via the reticulospinal tract and terminates on propriospinal neurons (PNs) located at
C3/4 in the spinal cord (Alstermark et al., 2007). PNs project to alpha motoneurons ( $\alpha \mathrm{MNs}$ ) innervating muscles involved in specific tasks so movements can be rapidly generated and modified as necessary (Pierrot-Deseilligny and Burke, 2005). Our hypothesis is that neural inputs from the CRPP are integrated by PNs with those from the disynaptic (indirect) portion of the contralateral corticospinal tract. As a result, descending inputs from both hemispheres shape the final motor command reaching $\alpha \mathrm{MNs}$ innervating upper limb musculature for optimal movement control.

Up-regulation of contralesional motor cortex excitability and the CRPP pathway may be important for paretic arm function after stroke (Turton et al., 1996; Netz et al., 1997; Alagona et al., 2001; Lewis et al., 2004; Misawa et al., 2008), particularly in poorly recovered patients (Turton et al., 1996; Netz et al., 1997; Gerloff et al., 1998; Caramia et al., 2000; Trompetto et al., 2000; Lewis and Perreault, 2007; Misawa et al., 2008). The degree of reorganization toward contralesional hemisphere control may depend on the residual integrity of white matter tracts from the ipsilesional hemisphere (Ward et al., 2006, 2007; Stinear et al., 2008; Grefkes and Fink, 2011). The neurophysiological model proposed here explains how increased excitability of the CRPP disrupts the normal cM1-iM1 balance of descending inputs reaching C3/4 PNs. In patients with a relatively intact ipsilesional corticospinal tract, up-regulation of the CRPP pathway would interfere with descending commands to PNs from the ipsilesional cortex. The model also accounts for why the CRPP is integral to residual function 
when the ipsilesional corticospinal tract is severely compromised. In these patients the CRPP may be the only intact descending pathway from cortex to spinal cord, and therefore of particular importance for their motor recovery.

Finally, a contribution by contralesional M1 to upper limb motor control via the CRPP has implications for NBS protocols aimed at improving rehabilitation of the paretic upper limb after stroke. The proposed model shows that contralesional M1 suppression after NBS may affect stroke patients differently depending on the severity of damage to the ipsilesional corticospinal tract and the degree of up-regulation of the contralesional CRPP. Studies that have included more severely impaired patients seem to indicate paretic upper limb motor performance is degraded by contralesional M1 NBS (Ackerley et al., 2010; Theilig et al., 2011; Bradnam et al., 2012). We propose that NBS protocols that aim to suppress contralesional M1 may be contraindicated for some patients. We argue that NBS is not a "one size fits all" solution for recovery after stroke, but that it can be tailored to individual patients based on neurophysiological and clinical biomarkers that are relative easy to obtain (Stinear et al., 2007, 2012; Jang et al., 2010; Kwon et al., 2011; Riley et al., 2011).

\section{A NEUROPHYSIOLOGICAL MODEL FOR IPSILATERAL UPPER LIMB CONTROL}

Many tasks performed with one hand are challenging and require precise co-contraction of multiple muscles across the upper limb. Skilled and complex unimanual movements are accompanied by an increase in iM1 excitability (Hummel et al., 2003; Verstynen et al., 2005; Morishita et al., 2011; Uehara et al., 2011), potentially by modulation of transcallosal projections (Perez and Cohen, 2008; Morishita et al., 2011; Uehara et al., 2011)_ENREF_8 or by increased transmission through ipsilateral projections to spinal $\alpha \mathrm{MNs}$ (Gerloff et al., 1998). Using Transcranial Magnetic Stimulation (TMS), iM1 has been found to contribute to sequential movement timing and selective activation of proximal and distal muscles in healthy adults (Gerloff et al., 1998; Carey et al., 2006; Davare et al., 2007; Duque et al., 2008; Bradnam et al., 2010; McCambridge et al., 2011). It appears that muscle coordination for precise and skilled unimanual tasks requires activation of both cortical hemispheres. The neurophysiological model of bilateral motor control from cM1 and iM1 is illustrated schematically in Figure 1. Both cortical hemispheres have direct and indirect projections to $\alpha \mathrm{MNs}$ in the spinal cord (Kuypers, 1964; Brinkman and Kuypers, 1973). From cM1, the corticospinal tract descends in the internal capsule to the brainstem and onto the spinal cord where the monosynaptic (direct) portion terminates onto $\alpha \mathrm{MNs}$ in the ventral horn (Fries et al., 1993). The disynaptic (indirect) portion of the tract originates from distinct populations of neurons in $\mathrm{cM} 1$ and descends alongside the rubrospinal and tectospinal tracts to converge onto cervical PNs (Alstermark et al., 2007; Lemon, 2008). Ipsilateral descending motor control is also mediated by direct and indirect pathways. From iM1, approximately $10-15 \%$ of the corticospinal fibers are uncrossed and project directly to $\alpha \mathrm{MNs}$ in the ipsilateral spinal cord. The indirect ipsilateral descending tract is the CRPP. The proposed anatomical pathway is as follows. After traversing the internal capsule separately, descending projections from the ipsilateral premotor cortex and iM1 terminate on reticular neurons

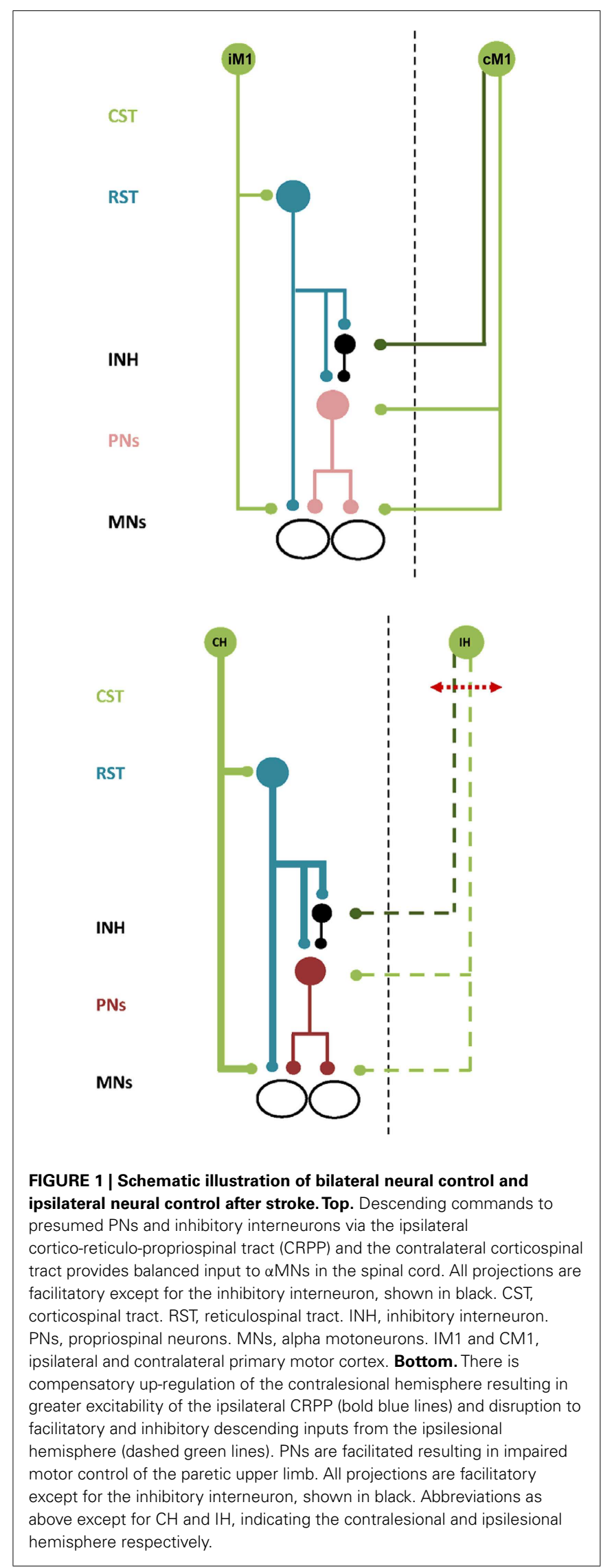


in the brainstem that give rise to the reticulospinal descending tracts (Andrews et al., 1973; Catsman-Berrevoets and Kuypers, 1976). The reticulospinal projections descend in the spinal cord as inhibitory (lateral) and excitatory (medial) tracts, terminating on medial motor nuclei in the spinal cord to innervate axial and proximal limb $\alpha$ MNs (Kuypers, 1964; Brinkman and Kuypers, 1973). In the non-human primate, reticulospinal tracts provide bilateral innervation to muscles of the proximal upper limb (Davidson and Buford, 2004, 2006; Davidson et al., 2007).

In the cat and non-human primate reticulospinal projections converge onto PNs (Brinkman and Kuypers, 1973; Illert et al., 1977, 1981; Alstermark et al., 1984; Boudrias et al., 2010) in addition to $\alpha \mathrm{MNs}$. PNs project to $\alpha \mathrm{MNs}$ innervating agonist proximal and distal muscles across the upper limb and to Ia inhibitory interneurons controlling $\alpha \mathrm{MNs}$ of antagonist muscles (Tantisira et al., 1996). Descending and ascending inhibitory control over PNs facilitates fine-tuning and precise shaping of motor commands prior to their execution by $\alpha M N s$. Behavioral studies in the cat show that partially transecting the spinal cord at C3-4 induces dysmetria and discoordination of the forelimb (Alstermark et al., 2007). In humans, the cervical propriospinal system is a candidate neural network underpinning muscle synergies for upper limb reaching (Pierrot-Deseilligny and Burke, 2005). Task-specific use of the hand disinhibits PNs to activate proximal wrist and shoulder muscle synergies important for limb stabilization (Iglesias et al., 2007; Roberts et al., 2008; Giboin et al., 2012). According to our hypothesis, descending commands from the CRPP are integrated with those from cM1 via the disynaptic (indirect) corticospinal tract within presumed C3/4 PNs. The balance of descending facilitation and inhibition from the two hemispheres dictates overall spinal PN excitability and subsequent activation of $\alpha M N s$. There is evidence in humans for an ipsilateral pathway from iM1 to spinal PNs in support of our model. c-tDCS applied to iM1 suppressed both facilitatory and inhibitory projections to ipsilateral PNs in the spinal cord of healthy adults (Bradnam et al., 2011), although involvement of the reticulospinal tract cannot be confirmed from this experiment. It also appears possible to indirectly modulate both upper and lower limb PN circuits using NBS over M1 (Bradnam et al., 2011; Roche et al., 2011,2012). ENREF_31 Future experiments may further elucidate how PNs integrate bilateral descending commands from motor cortex and the role of the cervical propriospinal system in complex upper limb tasks in humans.

Inhibitory projections to PNs may originate in premotor cortex rather than M1 (see Figure 1, top panel). This is important as descending tracts from premotor cortex are separated from those of M1 within the internal capsule. White matter tracts from premotor cortex descend in the genu and anterior portion of the posterior limb of the internal capsule, while those from M1 descend in the posterior limb itself (Fries et al., 1993). Therefore, the location of a stroke lesion in the internal capsule would have a significant impact on the residual ability of ipsilesional M1 to provide inhibitory modulation of PNs and $\alpha \mathrm{MNs}$.

\section{NEUROPHYSIOLOGICAL MODEL OF IPSILATERAL UPPER LIMB CONTROL AFTER STROKE}

After stroke there can be an increase in motor cortex excitability in the contralesional hemisphere and reduced excitability of the ipsilesional hemisphere. This view of interhemispheric imbalance of the motor system after stroke occurs via transcallosal inhibitory pathways between the two hemispheres (Shimizu et al., 2002; Murase et al., 2004; Ward et al., 2006, 2007; Grefkes et al., 2008; Ameli et al., 2009; Grefkes and Fink, 2011). Within the first 2 weeks after stroke contralesional hemispheric excitability can increase in patients with severe deficits (Rehme et al., 2011). The degree of interhemispheric imbalance in excitability and motor impairment may be negatively correlated with the residual integrity of the ipsilesional corticospinal tract after the stroke (Grefkes and Fink, 2011). This is supported by evidence that contralesional hemisphere activity is enhanced in patients with more extensive ipsilesional corticospinal tract disruption and greater upper limb impairment (Johansen-Berg et al., 2002; Ward et al., 2003, 2006, 2007; Lotze et al., 2006; Stinear et al., 2008). In well-recovered patients contralesional hemisphere excitability decreases over time (Stinear et al., 2008). In severely affected patients, contralesional hemisphere excitability gradually increases as motor function recovers, indicating reorganization toward the contralesional hemisphere (Stinear et al., 2008). This increase in contralesional M1 excitability is also marked by facilitation of ipsilateral descending projections to paretic upper limb muscles (Turton et al., 1996; Netz et al., 1997; Caramia et al., 2000; Alagona et al., 2001; Lewis and Perreault, 2007).

Cortical reorganization following spinal cord transection consists of an early phase of bilateral M1 up-regulation, followed later by increased contralesional M1 and bilateral premotor excitability (Nishimura et al., 2007; Nishimura and Isa, 2009). Hand dexterity can be restored by intensive rehabilitation following transection of the contralateral corticospinal tract in brain or cervical cord but is accompanied by abnormal co-activation of distal and proximal muscles (Nishimura et al., 2009), in particular forearm and hand flexors (Zaaimi et al., 2012). Furthermore, these studies have demonstrated there is only a weak contribution of ipsilateral M1 to residual motor control of the paretic upper limb (Schmidlin et al., 2004; Zaaimi et al., 2012), indicating intact subcortical descending pathways are responsible for the observed motor recovery (Nardone et al., 2013). Together these studies support an emerging concept of reorganization via ipsilateral reticulospinal and propriospinal systems following disruption to contralateral corticospinal tract in line with our hypothesis.

A model for how the ipsilateral CRPP may influence upper limb recovery after stroke is illustrated schematically in Figure 1, bottom panel. The model demonstrates that the balance of cM1iM1 descending inputs reaching $\mathrm{C} 3 / 4$ PNs can be affected by the stroke lesion. Descending motor control by the ipsilesional hemisphere is reduced with disruption to the corticospinal tract. This is associated with an increase in excitability of contralesional M1 and descending output along the ipsilateral CRPP, leading to a worsening of upper limb impairment. Why might this occur? Ipsilesional white matter damage confined to the posterior limb of the internal capsule may spare the descending inhibitory projections originating in the premotor cortex that traverse the genu of the internal capsule and the ventral portion of the posterior internal capsule (Fries et al., 1993). A moderate increase in presumed PN excitability via the CRPP could be balanced by the residual inhibitory control over PNs from the 
ipsilesional hemisphere. In contrast, extensive damage to the ipsilesional descending pathways would compromise both excitatory and inhibitory projections to presumed PNs. With greater tract damage there would be little remaining ipsilesional inhibitory control to counterbalance the significant facilitation of presumed PNs via the contralesional CRPP. It is also possible that small lesions may affect the CRPP in isolation and give rise to abnormal synergistic control through altered input to PNs and inhibitory interneurons. This model of ipsilateral CRPP up-regulation is supported by experiments showing that excitability of circuits mediated by presumed PNs is increased after stroke (Mazevet et al., 2003; Stinear and Byblow, 2004), suggesting up-regulation of these indirect descending pathways when the corticospinal tract is compromised.

The model predicts that contralesional CRPP influences upper limb function negatively to positively along a continuum that depends on ipsilesional white matter tract integrity. In patients with minimal damage to the corticospinal tract, the contralesional CRPP may interfere with residual descending inputs from ipsilesional hemisphere, at the level of the spinal cord. Greater descending drive through the ipsilateral reticulospinal tract compared to contralateral corticospinal tract may result in aberrant recruitment of PNs and inhibitory interneurons. In the presence of extensive damage, the contralesional CRPP may provide the only descending cortical commands to reach the spinal cord. This pathway can only partially compensate for the loss of excitatory and inhibitory descending control from ipsilesional M1. The loss of tonic inhibition over $\alpha \mathrm{MNs}$, and aberrant control of PNs, might explain the emergence of abnormal muscle synergies and spasticity in the paretic upper limb in more severely impaired patients after stroke. This model highlights the importance of the contralesional CRPP pathway for residual control over the paretic upper limb, which may have relevance for the use of NBS protocols that aim to suppress the contralesional M1.

\section{IMPLICATIONS FOR NON-INVASIVE BRAIN STIMULATION}

Non-invasive brain stimulation may be a useful adjuvant to rebalance motor cortex excitability after stroke, with the aim of improving motor function of the paretic upper limb during recovery (Hummel et al., 2008). Using NBS to directly facilitate ipsilesional M1 can improve paretic hand function by increasing excitability of descending projections to $\alpha \mathrm{MNs}$ (Fregni et al., 2005; Mansur et al., 2005; Takeuchi et al., 2005; Boggio et al., 2007; Nowak et al., 2008; Ackerley et al., 2010; Kim et al., 2010; Stagg et al., 2012). Conversely, NBS to suppress excitability of contralesional M1 may restore the balance of hemispheric excitability and also enhance corticomotor drive from ipsilesional M1 to the paretic upper limb (Takeuchi et al., 2005; Suppa et al., 2008; Grefkes et al., 2010). Improvements in hand function have been reported in studies using NBS to suppress contralesional M1 (Fregni et al., 2005; Boggio et al., 2007; Dafotakis et al., 2008; Nowak et al., 2008; Grefkes et al., 2010; Kim et al., 2010), however many studies have examined relatively well-recovered patients. In patients with more severe impairment suppression of the contralesional M1 has been equivocal for improving upper limb function (Ackerley et al., 2010; Theilig et al., 2011; Bradnam et al., 2012; Talelli et al., 2012). The prevailing view of interhemispheric imbalance of motor cortex excitability after stroke, and use of NBS to suppress contralesional M1 to redress imbalance, has not considered effects on output pathways other than transcallosal projections. Our model proposes that suppression of contralesional M1 can reduce excitability of the ipsilateral CRPP (Bradnam et al., 2011), and therefore the use of NBS for suppressing contralesional M1 should be considered carefully. The effects of suppressing contralesional M1 may be quite different for mildly impaired (Figures 2A,B) versus moderate to severely impaired (Figures 2C,D) patients, as illustrated schematically in Figure 2. In a study of patients with upper limb weakness after subcortical stroke, c-tDCS improved paretic proximal upper limb motor control in mildly impaired patients but degraded control in moderate to severely impaired patients (Bradnam et al., 2012). The residual structural integrity of the ipsilesional corticospinal tract was compromised in patients for whom suppression of the contralesional M1 was detrimental. It is conceivable that these patients rely more strongly on compensatory up-regulation of the contralesional M1 and CRPP. Suppression of contralesional M1 may reduce residual descending drive to $\alpha \mathrm{MNs}$, further degrading paretic upper limb motor control. Conversely, for mildly impaired patients, suppressing contralesional M1 may reduce interhemispheric inhibition of ipsilesional M1 leading to improved function of the paretic upper limb as described previously (Fregni et al., 2005; Boggio et al., 2007; Dafotakis et al., 2008; Nowak et al., 2008; Grefkes et al., 2010; Kim et al., 2010). Contralesional M1 suppression also decreases excitability of the contralesional CRPP and could therefore reduce interference between descending inputs to the cord from intact ipsilesional descending pathways and the contralesional CRPP.

This idea has several practical implications. First, measuring the effects of NBS on corticomotor excitability in contralateral distal hand muscles does not completely capture all the effects on descending pathways that reach spinal motoneurons. Second, suppression of contralesional M1 may be contraindicated in more severely affected stroke patients (Bradnam et al., 2012). Third, the proposed CRPP model supports the emerging view that NBS neuromodulation should be tailored individually for patients after stroke based on impairment level (at the chronic stage) (Cramer, 2010; Stinear, 2010; Bradnam et al., 2012) and perhaps, based on the residual capacity of their descending motor pathways (at the sub-acute stage). Further research examining the full range of neurophysiological effects induced by NBS seems warranted. For example, it may be advantageous to use NBS to either facilitate or attenuate contralesional hemisphere excitability based on the degree of damage to the ipsilesional descending pathways. This remains to be determined.

How might individualization of NBS be implemented to improve post-stroke rehabilitation? The presence or absence of motor evoked potentials from TMS and measures of structural integrity derived from diffusion-weighted imaging (DWI) provide objective information about corticospinal tract integrity. TMS and DWI can be used in combination with clinical measures to predict the potential for recovery of function with rehabilitation (Stinear et al., 2007, 2012; Jang et al., 2010; Kwon et al., 2011; Riley et al., 2011). TMS and DWI could also determine which patients are suitable for suppressive NBS to contralesional M1. A recent study indicated that patients with moderate damage to the 


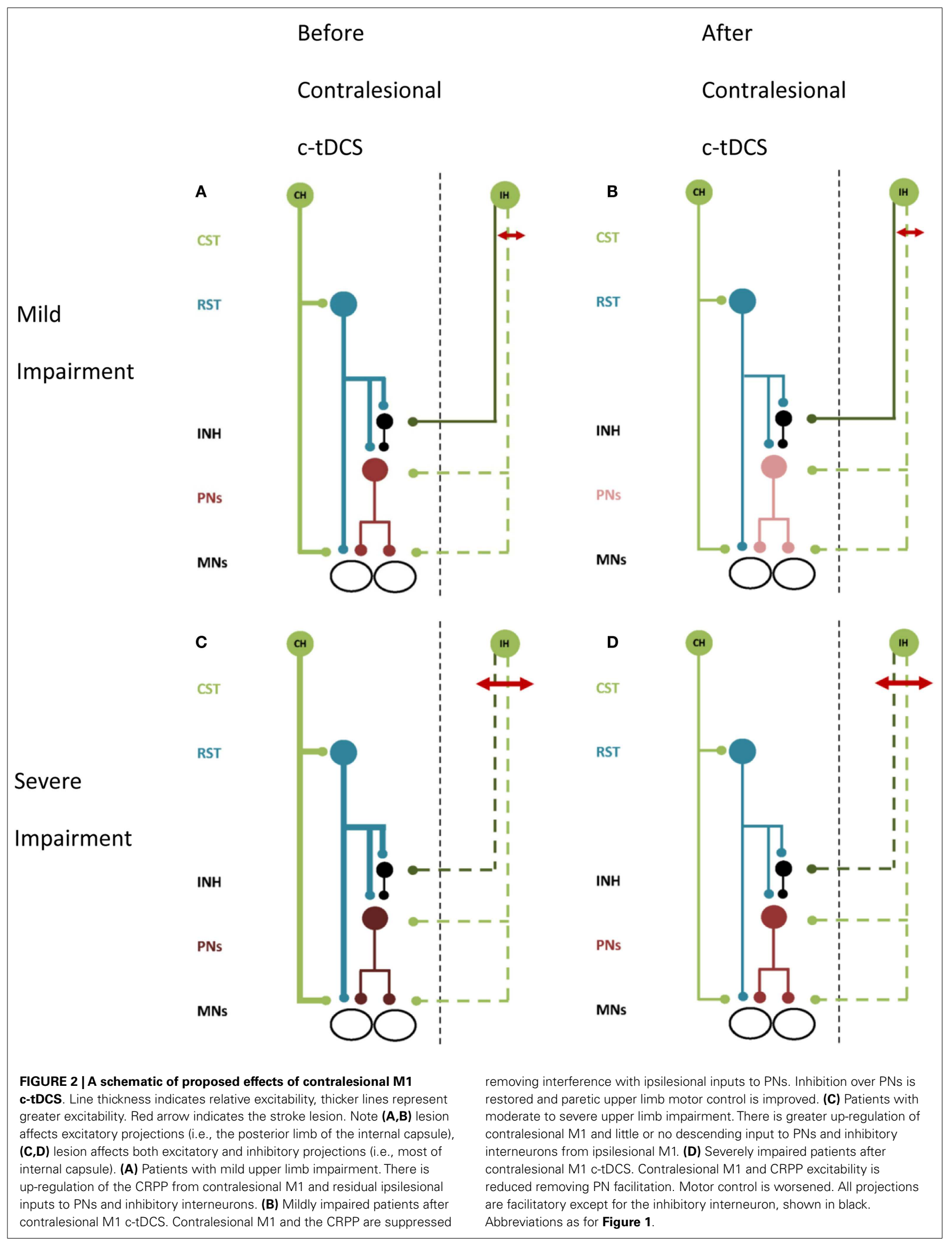


internal capsule did not have a positive response to contralesional M1 c-tDCS (Bradnam et al., 2012). Patients in whom motor control was improved by NBS were characterized by the presence of responses to TMS from stimulation of ipsilesional M1, and low impairment levels or no upper limb spasticity as assessed by clinical scales. These relatively simple measures could be used to help decide whether a given NBS protocol is appropriate for individual patients.

\section{CONCLUSION}

It is becoming increasingly clear that skilled function of the upper limb relies on the balance of excitability of $\mathrm{cM} 1$ and $\mathrm{iM} 1$ in healthy adults. In this article we propose a neurophysiological model of ipsilateral neural control of the proximal upper limb via the CRPP.

\section{REFERENCES}

Ackerley, S. J., Stinear, C. M., Barber, P. A., and Byblow, W. D. (2010). Combining theta burst stimulation with training after subcortical stroke. Stroke 41, 1568-1572.

Alagona, G., Delvaux, V., Gerard, P., De Pasqua, V., Pennisi, G., Delwaide, P. J., et al. (2001). Ipsilateral motor responses to focal transcranial magnetic stimulation in healthy subjects and acute-stroke patients. Stroke 32, 1304-1309.

Alstermark, B., Isa, T., Pettersson, L. G., and Sasaki, S. (2007). The C3-C4 propriospinal system in the cat and monkey: a spinal pre-motoneuronal centre for voluntary motor control. Acta Physiol. (Oxf.) 189, 123-140.

Alstermark, B., Lundberg, A., and Sasaki, S. (1984). Integration in descending motor pathways controlling the forelimb in the cat. 12. Interneurones which may mediate descending feed-forward inhibition and feed-back inhibition from the forelimb to C3-C4 propriospinal neurones. Exp. Brain Res. 56, 308-322.

Ameli, M., Grefkes, C., Kemper, F., Riegg, F. P., Rehme, A. K., Karbe, H., et al. (2009). Differential effects of high-frequency repetitive transcranial magnetic stimulation over ipsilesional primary motor cortex in cortical and subcortical middle cerebral artery stroke. Ann. Neurol. 66, 298-309.

Andrews, C., Knowles, L., and Hancock, J. (1973). Control of the tonic vibration reflex by the brain stem reticular formation in the cat. J. Neurol. Sci. 18, 217-226.

Boggio, P. S., Nunes, A., Rigonatti, S. P., Nitsche, M. A., Pascual-Leone, A., and Fregni, F. (2007). Repeated sessions of noninvasive brain DC stimulation is associated with motor function improvement in stroke patients. Restor. Neurol. Neurosci. 25, 123-129.

Boudrias, M. H., Mcpherson, R. L., Frost, S. B., and Cheney, P. D. (2010). Output properties and organization of the forelimb representation of motor areas on the lateral aspect of the hemisphere in rhesus macaques. Cereb. Cortex 20, 169-186.

Bradnam, L. V., Stinear, C. M., Barber, P. A., and Byblow, W. D. (2012). Contralesional hemisphere control of the proximal paretic upper limb following stroke. Cereb. Cortex 22, 2662-2671.

Bradnam, L. V., Stinear, C. M., and Byblow, W. D. (2010). Theta burst stimulation of human primary motor cortex degrades selective muscle activation in the ipsilateral arm. J. Neurophysiol. 104, 2594-2602.

Bradnam, L. V., Stinear, C. M., and Byblow, W. D. (2011). Cathodal transcranial direct current stimulation suppresses ipsilateral projections to presumed propriospinal neurons of the proximal upper limb. J. Neurophysiol. 105, 2582-2589.

Brinkman, J., and Kuypers, H. G. (1973). Cerebral control of contralateral and ipsilateral arm, hand and finger movements in the split-brain rhesus monkey. Brain 96, 653-674.

Caramia, M. D., Palmieri, M. G., Giacomini, P., Iani, C., Dally, L., and Silvestrini, M. (2000). Ipsilateral activation of the unaffected motor cortex in patients with hemiparetic stroke. Clin. Neurophysiol. 111, 1990-1996.

Carey, J. R., Fregni, F., and PascualLeone, A. (2006). rTMS combined with motor learning training in healthy subjects. Restor. Neurol. Neurosci. 24, 191-199.

Catsman-Berrevoets, C. E., and Kuypers, H. G. (1976). Cells of origin of cortical projections to dorsal column nuclei, spinal cord and

The model highlights the importance of understanding how the degree of compensatory activity in the contralesional hemisphere after stroke contributes to paretic upper limb function across a range of impairment levels. It is proposed that NBS protocols are not "one size fits all" and should be carefully selected based on individual patient characteristics. Research has targeted the development of structural and functional biomarkers that can be combined with clinical tests to allow NBS to be individually prescribed as an adjuvant to therapy after stroke. These methods of patient stratification based on objective measures of impairment should be used to determine the optimum intervention in future studies of NBS in stroke. Individualization may increase effectiveness of NBS in clinical trials and expedite translation from research laboratory to the clinical setting.

bulbar medial reticular formation in the rhesus monkey. Neurosci. Lett. 3, 245-252.

Chen, R., Gerloff, C., Hallett, M., and Cohen, L. G. (1997). Involvement of the ipsilateral motor cortex in finger movements of different complexities. Ann. Neurol. 41, 247-254.

Cramer, S. C. (2010). Stratifying patients with stroke in trials that target brain repair. Stroke 41, S114-116. Dafotakis, M., Grefkes, C., Eickhoff, S. B., Karbe, H., Fink, G. R., and Nowak, D. A. (2008). Effects of rTMS on grip force control following subcortical stroke. Exp. Neurol. 211, 407-412.

Davare, M., Duque, J., Vandermeeren, Y., Thonnard, J. L., and Olivier, E. (2007). Role of the ipsilateral primary motor cortex in controlling the timing of hand muscle recruitment. Cereb. Cortex 17, 353-362.

Davidson, A. G., and Buford, J. A. (2004). Motor outputs from the primate reticular formation to shoulder muscles as revealed by stimulustriggered averaging. J. Neurophysiol. 92, 83-95.

Davidson, A. G., and Buford, J. A (2006). Bilateral actions of the reticulospinal tract on arm and shoulder muscles in the monkey: stimulus triggered averaging. Exp. Brain Res. 173, 25-39.

Davidson, A. G., Schieber, M. H., and Buford, J. A. (2007). Bilateral spiketriggered average effects in arm and shoulder muscles from the monkey pontomedullary reticular formation. J. Neurosci. 27, 8053-8058.

Duque, J., Mazzocchio, R., Stefan, K., Hummel, F., Olivier, E., and Cohen, L. G. (2008). Memory formation in the motor cortex ipsilateral to a training hand. Cereb. Cortex 18 , 1395-1406.

Fregni, F., Boggio, P. S., Mansur, C. G., Wagner, T., Ferreira, M. J., Lima, M. C., et al. (2005). Transcranial direct current stimulation of the unaffected hemisphere in stroke patients. Neuroreport 16, 1551-1555.

Fries, W., Danek, A., Scheidtmann, K., and Hamburger, C. (1993) Motor recovery following capsular stroke. Role of descending pathways from multiple motor areas. Brain 116(Pt 2), 369-382.

Gerloff, C., Cohen, L. G., Floeter, M. K., Chen, R., Corwell, B., and Hallett, M. (1998). Inhibitory influence of the ipsilateral motor cortex on responses to stimulation of the human cortex and pyramidal tract. J. Physiol. (Lond.) 510(Pt 1), 249-259.

Giboin, L. S., Lackmy-Vallee, A., Burke, D., and Marchand-Pauvert, V. (2012). Enhanced propriospinal excitation from hand muscles to wrist flexors during reach-to-grasp in humans. J. Neurophysiol. 107, 532-543.

Grefkes, C., and Fink, G. R. (2011). Reorganization of cerebral networks after stroke: new insights from neuroimaging with connectivity approaches. Brain 134, 1264-1276.

Grefkes, C., Nowak, D. A., Eickhoff, S. B., Dafotakis, M., Kust, J., Karbe, H., et al. (2008). Cortical connectivity after subcortical stroke assessed with functional magnetic resonance imaging. Ann. Neurol. 63, 236-246.

Grefkes, C., Nowak, D. A., Wang, L. E., Dafotakis, M., Eickhoff, S. B., and Fink, G. R. (2010). Modulating cortical connectivity in stroke patients by rTMS assessed with fMRI and dynamic causal modeling. Neuroimage 50, 233-242.

Hummel, F., Celnik, P., Pascual-Leone, A., Frengi, F., Byblow, W. D., Butefisch, C. M., et al. (2008). Controversy: noninvasive and invasive cortical stimulation show efficacy in treating stroke patients. Brain Stimulat. 1, 370-382. 
Hummel, F., Kirsammer, R., and Gerloff, C. (2003). Ipsilateral cortical activation during finger sequences of increasing complexity: representation of movement difficulty or memory load? Clin. Neurophysiol. 114, 605-613.

Iglesias, C., Marchand-Pauvert, V., Lourenco, G., Burke, D., and PierrotDeseilligny, E. (2007). Task-related changes in propriospinal excitation from hand muscles to human flexor carpi radialis motoneurones. J. Physiol. (Lond.) 582, 1361-1379.

Illert, M., Jankowska, E., Lundberg, A., and Odutola, A. (1981). Integration in descending motor pathways controlling the forelimb in the cat. 7 . Effects from the reticular formation on C3-C4 propriospinal neurones. Exp. Brain Res. 42, 269-281.

Illert, M., Lundberg, A., and Tanaka, R. (1977). Integration in descending motor pathways controlling the forelimb in the cat. 3. Convergence on propriospinal neurones transmitting disynaptic excitation from the corticospinal tract and other descending tracts. Exp. Brain Res. 29, 323-346.

Isa, T., Ohki, Y., Seki, K., and Alstermark, B. (2006). Properties of propriospinal neurons in the $\mathrm{C} 3-\mathrm{C} 4$ segments mediating disynaptic pyramidal excitation to forelimb motoneurons in the macaque monkey. J. Neurophysiol. 95, 3674-3685.

Jang, S. H., Ahn, S. H., Sakong, J., Byun, W. M., Choi, B. Y., Chang, C. H., et al. (2010). Comparison of TMS and DTT for predicting motor outcome in intracerebral hemorrhage. J. Neurol. Sci. 290, 107-111.

Johansen-Berg, H., Rushworth, M. F., Bogdanovic, M. D., Kischka, U., Wimalaratna, S., and Matthews, P. M. (2002). The role of ipsilateral premotor cortex in hand movement after stroke. Proc. Natl. Acad. Sci. U.S.A. 99, 14518-14523.

Kim, D. Y., Lim, J. Y., Kang, E. K., You, D. S., Oh, M. K., Oh, B. M., et al. (2010). Effect of transcranial direct current stimulation on motor recovery in patients with subacute stroke. Am. J. Phys. Med. Rehabil. 89, 879-886.

Kuypers, H. G. (1964). The descending pathways to the spinal cord, their anatomy and function. Prog. Brain Res. 11, 178-202.

Kwon, Y. H., Son, S. M., Lee, J., Bai, D. S., and Jang, S. H. (2011). Combined study of transcranial magnetic stimulation and diffusion tensor tractography for prediction of motor outcome in patients with corona radiata infarct. J. Rehabil. Med. 43, 430-434.

Lee, M., Hinder, M. R., Gandevia, S. C., and Carroll, T. J. (2010). The ipsilateral motor cortex contributes to cross-limb transfer of performance gains after ballistic motor practice. J. Physiol. (Lond.) 588, 201-212.

Lemon, R. N. (2008). Descending pathways in motor control. Annu. Rev. Neurosci. 31, 195-218.

Lewis, G. N., and Perreault, E. J. (2007). Side of lesion influences bilateral activation in chronic, post-stroke hemiparesis. Clin. Neurophysiol. 118, 2050-2062.

Lewis, G. N., Polych, M. A., and Byblow, W. D. (2004). Proposed cortical and sub-cortical contributions to the long-latency stretch reflex in the forearm. Exp. Brain Res. 156, 72-79.

Lotze, M., Markert, J., Sauseng, P., Hoppe, J., Plewnia, C., and Gerloff, C. (2006). The role of multiple contralesional motor areas for complex hand movements after internal capsular lesion. J. Neurosci. 26, 6096-6102.

Mansur, C. G., Fregni, F., Boggio, P. S., Riberto, M., Gallucci-Neto, J., Santos, C. M., et al. (2005). A sham stimulation-controlled trial of rTMS of the unaffected hemisphere in stroke patients. Neurology 64, 1802-1804.

Mazevet, D., Meunier, S., PradatDiehl, P., Marchand-Pauvert, V., and Pierrot-Deseilligny, E. (2003). Changes in propriospinally mediated excitation of upper limb motoneurons in stroke patients. Brain 126, 988-1000.

McCambridge, A. B., Bradnam, L. V., Stinear, C. M., and Byblow, W. D. (2011). Cathodal transcranial direct current stimulation of the primary motor cortex improves selective muscle activation in the ipsilateral arm. J. Neurophysiol. 105, 2937-2942.

Misawa, S., Kuwabara, S., Matsuda, S. Honma, K., Ono, J., and Hattori, T. (2008). The ipsilateral corticospinal tract is activated after hemiparetic stroke. Eur. J. Neurol. 15, 706-711.

Morishita, T., Ninomiya, M., Uehara, K., and Funase, K. (2011). Increased excitability and reduced intracortical inhibition in the ipsilateral primary motor cortex during a finemotor manipulation task. Brain Res. 1371, 65-73.

Muellbacher, W., Facchini, S., Boroojerdi, B., and Hallett, M. (2000). Changes in motor cortex excitability during ipsilateral hand muscle activation in humans. Clin. Neurophysiol. 111, 344-349.

Murase, N., Duque, J., Mazzocchio, R., and Cohen, L. G. (2004). Influence of interhemispheric interactions on motor function in chronic stroke. Ann. Neurol. 55, 400-409.

Nardone, R., Holler, Y., Brigo, F., Seidl, M., Christova, M., Bergmann, J., et al. (2013). Functional brain reorganization after spinal cord injury: systematic review of animal and human studies. Brain Res. 1504, 58-73.

Netz, J., Lammers, T., and Homberg, V. (1997). Reorganization of motor output in the non-affected hemisphere after stroke. Brain 120(Pt 9), 1579-1586.

Nishimura, Y., and Isa, T. (2009). Compensatory changes at the cerebral cortical level after spinal cord injury. Neuroscientist 15, 436-444.

Nishimura, Y., Morichika, Y., and Isa, T. (2009). A subcortical oscillatory network contributes to recovery of hand dexterity after spinal cord injury. Brain 132, 709-721.

Nishimura, Y., Onoe, H., Morichika, Y. Perfiliev, S., Tsukada, H., and Isa T. (2007). Time-dependent central compensatory mechanisms of finger dexterity after spinal cord injury. Science 318, 1150-1155.

Nowak, D. A., Grefkes, C., Dafotakis, M., Eickhoff, S., Kust, J. Karbe, H., et al. (2008). Effects of low-frequency repetitive transcranial magnetic stimulation of the contralesional primary motor cortex on movement kinematics and neural activity in subcortical stroke. Arch. Neurol. 65, 741-747.

Perez, M. A., and Cohen, L. G. (2008). Mechanisms underlying functional changes in the primary motor cortex ipsilateral to an active hand. $J$. Neurosci. 28, 5631-5640.

Perez, M. A., and Cohen, L. G. (2009). Scaling of motor cortical excitability during unimanual force generation. Cortex 45, 1065-1071.

Pierrot-Deseilligny, E., and Burke, D. (2005). The Circuitry of the Human Spinal Cord. Its Role in Motor Control and Movement Disorders. Cambridge: Cambridge University Press.

Rehme, A. K., Fink, G. R., Von Cramon, D. Y., and Grefkes, C. (2011). The role of the contralesional motor cortex for motor recovery in the early days after stroke assessed with longitudinal FMRI. Cereb. Cortex 21, 756-768.

Riley, J. D., Le, V., Der-Yeghiaian, L. See, J., Newton, J. M., Ward, N. S., et al. (2011). Anatomy of stroke injury predicts gains from therapy. Stroke 42, 421-426.

Roberts, L. V., Stinear, C. M., Lewis, G. N., and Byblow, W. D. (2008). Task-dependent modulation of propriospinal inputs to human shoulder. J. Neurophysiol. 100, 2109-2114.

Roche, N., Lackmy, A., Achache, V., Bussel, B., and Katz, R. (2011). Effects of anodal transcranial direct current stimulation over the leg motor area on lumbar spinal network excitability in healthy subjects. J. Physiol. (Lond.) 589, 2813-2826.

Roche, N., Lackmy, A., Achache, V., Bussel, B., and Katz, R. (2012). Effects of anodal tDCS on lumbar propriospinal system in healthy subjects. Clin. Neurophysiol. 123, 1027-1034.

Schmidlin, E., Wannier, T., Bloch, J., and Rouiller, E. M. (2004). Progressive plastic changes in the hand representation of the primary motor cortex parallel incomplete recovery from a unilateral section of the corticospinal tract at cervical level in monkeys. Brain Res. 1017, 172-183.

Shimizu, T., Hosaki, A., Hino, T., Sato, M., Komori, T., Hirai, S., et al. (2002). Motor cortical disinhibition in the unaffected hemisphere after unilateral cortical stroke. Brain 125 , 1896-1907.

Sohn, Y. H., Jung, H. Y., Kaelin-Lang, A., and Hallett, M. (2003). Excitability of the ipsilateral motor cortex during phasic voluntary hand movement. Exp. Brain Res. 148, 176-185.

Stagg, C. J., Bachtiar, V., O'shea, J., Allman, C., Bosnell, R. A., Kischka, U., et al. (2012). Cortical activation changes underlying stimulation-induced behavioural gains in chronic stroke. Brain 135, 276-284.

Stinear, C. (2010). Prediction of recovery of motor function after stroke. Lancet Neurol. 9, 1228-1232.

Stinear, C. M., Barber, P. A., Coxon, J. P., Fleming, M. K., and Byblow, W. D. (2008). Priming the motor system enhances the effects of upper limb therapy in chronic stroke. Brain 131, 1381-1390.

Stinear, C. M., Barber, P. A., Petoe, M., Anwar, S., and Byblow, W. D. (2012). The PREP algorithm predicts potential for upper limb recovery after stroke. Brain 135, 2527-2535.

Stinear, C. M., Barber, P. A., Smale, P. R., Coxon, J. P., Fleming, M. K., and Byblow, W. D. (2007). 
Functional potential in chronic stroke patients depends on corticospinal tract integrity. Brain 130, 170-180.

Stinear, J. W., and Byblow, W. D. (2004). The contribution of cervical propriospinal premotoneurons in recovering hemiparetic stroke patients. J. Clin. Neurophysiol. 21, 426-434.

Suppa, A., Ortu, E., Zafar, N., Deriu, F., Paulus, W., Berardelli, A., et al. (2008). Theta burst stimulation induces after-effects on contralateral primary motor cortex excitability in humans. J. Physiol. (Lond.) 586, 4489-4500.

Takeuchi, N., Chuma, T., Matsuo, Y., Watanabe, I., and Ikoma, K. (2005). Repetitive transcranial magnetic stimulation of contralesional primary motor cortex improves hand function after stroke. Stroke 36, 2681-2686.

Talelli, P., Wallace, A., Dileone, M., Hoad, D., Cheeran, B., Oliver, R., et al. (2012). Theta burst stimulation in the rehabilitation of the upper limb: a semirandomized, placebo-controlled trial in chronic stroke patients. Neurorehabil. Neural Repair 26, 976-987.
Tantisira, B., Alstermark, B., Isa, T., Kummel, H., and Pinter, M. (1996). Motoneuronal projection pattern of single C3-C4 propriospinal neurones. Can. J. Physiol. Pharmacol. 74, 518-530.

Theilig, S., Podubecka, J., Bosl, K., Wiederer, R., and Nowak, D. A. (2011). Functional neuromuscular stimulation to improve severe hand dysfunction after stroke: does inhibitory rTMS enhance therapeutic efficiency? Exp. Neurol. 230, 149-155.

Trompetto, C., Assini, A., Buccolieri, A., Marchese, R., and Abbruzzese, G. (2000). Motor recovery following stroke: a transcranial magnetic stimulation study. Clin. Neurophysiol. 111, 1860-1867.

Turton, A., Wroe, S., Trepte, N., Fraser, C., and Lemon, R. N. (1996). Contralateral and ipsilateral EMG responses to transcranial magnetic stimulation during recovery of arm and hand function after stroke. Electroencephalogr. Clin. Neurophysiol. 101, 316-328.

Uehara, K., Morishita, T., and Funase, K. (2011). Excitability changes in the ipsilateral primary motor cortex during rhythmic contraction of finger muscles. Neurosci. Lett. 488 , 22-25.

Verstynen, T., Diedrichsen, J., Albert, N., Aparicio, P., and Ivry, R. B. (2005). Ipsilateral motor cortex activity during unimanual hand movements relates to task complexity. J. Neurophysiol. 93, 1209-1222.

Ward, N. S., Brown, M. M., Thompson, A. J., and Frackowiak, R. S. (2003). Neural correlates of outcome after stroke: a crosssectional fMRI study. Brain 126, 1430-1448.

Ward, N. S., Newton, J. M., Swayne, O. B., Lee, L., Frackowiak, R. S., Thompson, A. J., et al. (2007). The relationship between brain activity and peak grip force is modulated by corticospinal system integrity after subcortical stroke. Eur. J. Neurosci. 25, 1865-1873.

Ward, N. S., Newton, J. M., Swayne, O. B., Lee, L., Thompson, A. J., Greenwood, R. J., et al. (2006). Motor system activation after subcortical stroke depends on corticospinal system integrity. Brain 129 , 809-819.

Zaaimi, B., Edgley, S. A., Soteropoulos, D. S., and Baker, S. N.
(2012). Changes in descending motor pathway connectivity after corticospinal tract lesion in macaque monkey. Brain 135, 2277-2289.

Conflict of Interest Statement: The authors declare that the research was conducted in the absence of any commercial or financial relationships that could be construed as a potential conflict of interest.

Received: 22 January 2013; paper pending published: 04 April 2013; accepted: 23 April 2013; published online: 08 May 2013.

Citation: Bradnam LV, Stinear CM and Byblow WD (2013) Ipsilateral motor pathways after stroke: implications for non-invasive brain stimulation. Front. Hum. Neurosci. 7:184. doi: 10.3389/fnhum.2013.00184

Copyright (c) 2013 Bradnam, Stinear and Byblow. This is an open-access article distributed under the terms of the Creative Commons Attribution License, which permits use, distribution and reproduction in other forums, provided the original authors and source are credited and subject to any copyright notices concerning any third-party graphics etc. 